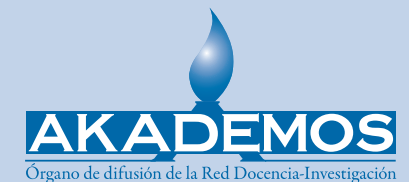

ISSN: 1995-4743

Año 11 Vol. 2, n. 29 Julio-Diciembre 2017

AKADEMOS es una revista semestral. De amplio espacio editorial, para la publicación de trabajos inéditos de investigación, articulos de análisis, reseñas y opinión, en los distintos tópicos de las ciencias, la tecnología, las artes y la cultura.

\section{La calidad de la Educación Artística} en la formación docente.

\section{Recorridos y desafíos}

Conversatorio con la Dra. Marta Eugenia Valle Contreras y la Mtr. Sara María Boulogne', moderado por el Lic. Mario Zetino²

Marta Eugenia Valle Contreras

Investigadora del Centro de Investigaciones en Ciencias y Humanidades, $\mathrm{CICH}$ de la Universidad Dr. José Matías Delgado. Dra. en Educación por la Universidad de Granada, España. Ejecuta el Programa de Investigaciones para el Fomento de la Educación Artística desde el 2008.

mevallec@ujmd.edu.sv
Palabras introductorias por el Dr. Oscar Picardo Joao ${ }^{3}$

Como todos lo sabemos, el docente es un factor determinante dentro del sistema educativo. Sin embargo, en nuestro medio, la profesión docente, en términos generales, es una profesión poco valorada socialmente, poco dignificada, y en nuestra historia, es una de las pocas cosas que se ha tocado en materia de reforma o transformación; se ha tratado de un modo muy superficial. Se ha reformado todo: se ha reformado el currículo, se han reformado libros de texto, las tecnologías... Cuanta cosa se pudo reformar se reformó; sin embargo, cuando se diseñó el programa de formación docente que está vigente, del año 97, a raíz del cierre de las escuelas normales, se creó un profesorado de nivel universitario, con pocos controles de calidad.

1 Licenciada en Artes Visuales. Coordinadora del Programa Nacional de Formación de Docentes en Servicio en Educación Artística (MINED-FEPADE).

2 Investigador del $\mathrm{CICH}$. Licenciado en Letras. Forma parte del equipo de investigación del Programa de Investigaciones para el Fomento de la Educación Artística desde el 2017.

3 Director del Centro de Investigaciones en Ciencias y Humanidades, $\mathrm{CICH}$ de la Universidad Dr. José Matías Delgado. 
Como decíamos en el conversatorio que tuvimos sobre currículum ${ }^{4}$, la pregunta es: ¿quién quiere ser docente en El Salvador, y por qué razón? Veamos a modo de ejemplo: ¿cuáles son los mecanismos para optar por esta carrera? Comparemos la docencia con la carrera médica, una comparación bastante válida si decimos que la formación docente es importante.

¿Por qué una carrera como la de un docente, un profesorado, se reduce a tres años, cuando un maestro de Educación Básica tiene la responsabilidad de enseñar Matemáticas, Ciencias, Lenguaje y Estudios Sociales? ¿Y por qué la carrera de médico tiene una amplitud y una profundidad de siete años, con las cuatro especialidades...? Lo ponemos como una especie de caricatura real. Inclusive, en algunas universidades, la docencia era el resguardo de aquellos aspirantes que no podían entrar a otras carreras. Aspiraban a ser abogados o médicos o ingenieros, pero cuando sus resultados de admisión no eran satisfactorios, pues les invitaban a que ingresaran aunque sea a la carrera de profesorado.

En síntesis —es una realidad-, la docencia es una profesión demasiado importante para que se haya tratado bajo los cánones actuales en materia de políticas educativas.

Esta tarde se va a conversar sobre Educación Artística, un área curricular que ha estado a la sombra de Matemáticas, Ciencias, Estudios Sociales y Lenguaje. La Educación Artística y Educación Física allí han estado arrinconadas. Algunos colegios, escuelas o institutos tienen la dicha de tener un pro- fesor medianamente formado y le dedican algún espacio a la educación artística, pero la gran mayoría no tiene espacio curricular para esta asignatura.

También hablamos de esto en anteriores conversatorios: sobre lo importante que es la formación estética, en las tres áreas que recomienda el currículo nacional: expresión plástica, artes escénicas y música. Y si bien representa un reto formativo tener un maestro de Educación Artística que tenga un dominio holístico, de todas las áreas, pues nos damos cuenta que ni una ni otra, ni música ni expresión plástica ni artes escénicas. Y tampoco las pruebas estandarizadas internacionales valoran esta asignatura. Lo que importa es matemáticas y ciencias. Las pruebas PISA, las pruebas de la OCDE, y casi todas las pruebas habidas y por haber, lo que miden es la estatura de un país por sus resultados en ciencias y matemáticas. De tal forma que generalmente a las autoridades ministeriales no les importa mucho si hay o no hay, y por eso estamos como estamos.

Es decir, parte de lo que cosechamos hoy en día, un tejido social desfigurado, con un nivel de descomposición bastante alto con el factor de las pandillas y el de la migración, tiene mucho que ver con lo que hemos hecho en estos veinte años de reformas. Veinte años que se han tragado miles de millones de dólares. Pese a todos los préstamos, pese a toda la cooperación, pese a todo lo

4 Conversatorio con el Dr. Oscar Picardo Joao, "Aportes del CICH a la teoría educativa", realizado el jueves 7 de septiembre. 
que se ha invertido, pues, aquí tenemos los resultados. Y parece que vamos a seguir cosechando lo mismo en el corto, mediano y largo plazo.

Lamentable no se vislumbran cambios de fondo; hay cambios cosméticos. Seguimos con ausencia de políticas de Estado, de políticas de gobierno. Cada cinco años se cambia todo. Primero fue el Plan Decenal, después el desafío de la Educación para el Nuevo Milenio, después fue el plan 20-21, después el plan Vamos a la Escuela, después el plan El Salvador Educado. Y en el 2019 seguramente tendremos otras locuras con el mismo 3\% del PIB. De tal forma que el pronóstico es un poco pesimista. Y como universidad y como sociedad, tenemos que ser un poco más exigentes.

Así que no quiero tomar más tiempo, y quiero invitarlos a que aprovechen y disfruten lo que tienen que decir Marta Eugenia, Sara y Mario: qué tenemos, para dónde vamos, cuáles son las expectativas y qué podemos esperar para lo que viene en materia de políticas públicas, sobre todo en la combinación de formación docente y Educación Artística. Agradezco el trabajo que ha hecho durante todos estos años Marta Eugenia, que se ha dedicado a formarse a sí misma, ya que sacó su doctorado, y a ayudar a otros en este campo, y se ha hecho una especialista reconocida, de tal forma que podemos decir que es una autoridad en la materia de Educación Artística, una de las pocas especialistas con doctorado en este campo. Y lo ha demostrado, además, con su participación en ponencias a nivel local, en países de la región y hasta en China, adonde fue invitada.

Así que gracias por los aportes, y vamos a disfrutar de este conversatorio.

\section{Conversatorio}

Mario Zetino (MZ): Buenas tardes. Es un gusto enorme para mí estar en este conversatorio con Marta Eugenia Valle y Sara Boulogne, quienes, como ya hemos podido escuchar, tienen una amplia y profunda trayectoria como artistas, educadoras e investigadoras. Y para comenzar este conversatorio, quisiera invitar a Marta Eugenia a que nos exponga introductoriamente el tema. Por favor, Marta Eugenia.

\section{Marta Eugenia Valle (MEV): Buenas} tardes. Un afectuoso saludo a quienes nos acompañan. Gracias por compartir este itinerario. Hoy queremos poner en perspectiva este tema, la educación artística, que no ha sido muy tocado en realidad en El Salvador.

Hemos estado hablando, como podemos ver en la fotografía [refiriéndose a una diapositiva], del alumnado al que nos debemos. Una imagen habla más que mil palabras. Observamos a estos estudiantes y ellos nos observan, expectantes de lo que nosotros podemos hacer por la educación en El Salvador.

Quisiera mostrarles, para hacer un encuadre antes de comenzar, lo que es el Programa de Investigación para el Fomento de la Educación Artística que se desarrolla en el CICH desde el 2008. 
Este programa tiene cuatro componentes. El primero es la investigación de campo; el segundo, el programa de desarrollo de productos derivados de la investigación; el tercero, el Observatorio de Cultura, Artes y Educación Artística, de donde se deriva esta publicación que es La Educación Artística en la Enseñanza Básica en El Salvador. Este producto es uno de los que ha incidido más, de lo que hemos aportado desde el Observatorio de Cultura, Artes y Educación Artística, para poner en agenda educativa nacional a la Educación Artística. Y el cuarto componente es el Programa de Formación Docente en Educación Artística, que tiene varias versiones. Por ahora estamos trabajando en educación continua.

El aporte ha sido bastante claro, y ha incidido en las políticas nacionales. Ahora que ya tenemos esa atención del ministerio, también hay que llamar la atención de la sociedad salvadoreña y de los tomadores de decisión sobre qué es lo que viene. Entonces, si queremos una educación artística de calidad en la enseñanza básica, ¿adónde tendríamos que poner los énfasis en este momento? Y esto es lo que estamos trabajando en el programa de formación docente. Sabemos que el techo de la calidad de la educación está en la formación de los docentes, por eso creemos que es fundamental que se refuerce esa área, cómo está recibiendo el maestro su formación en Educación Artística, antes de esperar resultados.

Así que este es el marco en el que vamos a platicar este día. Agradezco la presencia, para compartir, de Sara Boulogne; de Mario, que gentilmente nos acompaña como moderador, y que es también parte del equipo de investigación que tenemos en el Centro de Investigación en este tema, y ha formado parte de los procesos de formación con los maestros. Con él estamos trabajando en campo como parte del componente de formación docente [del programa de investigación]. Estamos haciendo validación pedagógica con un centro escolar de Talnique. Eso es lo que nos ocupa en este momento: una propuesta pedagógica en la que se fusiona el Enfoque Educativo Biocéntrico con la educación artística.

Creo que va a haber tiempo de que platiquemos un poquito más de las características de esta propuesta, pero por ahora los dejaría con este marco para que arranquemos.

MZ: Gracias, Marta Eugenia. Y como parte de este marco introductorio, Marta Eugenia nos quiere compartir un par de videos acerca de la Cumbre Mundial de Educación Artística a la que asistió como ponente el año pasado, en Hangzhou, China. [Pasa el video This is Arts. (Congreso Mundial de Educación Artística. Hangzhou, China, diciembre 2016. World Aliance for Arts Education, WAAE)].

Estas son unas introducciones para inspirarnos a hablar sobre el arte y la educación

5 Valle Contreras, Marta Eugenia. (2011). La Educación Artística en la Enseñanza Básica en El Salvador. Antiguo Cuscatlán: Centro de Investigaciones en Ciencias y Humanidades (CICH)/Editorial Delgado. Universidad Dr. José Matías Delgado. 
artística. Y quisiera comenzar retomando una de las frases del director de esta cumbre mundial, que dice que "El propósito de la educación artística es guiar a la niñez para que encuentre, aprecie y entienda la belleza, y a partir de allí pueda crear arte".

¿Qué me pueden decir de esto? ¿Es realmente este el propósito de la educación artística según lo entendemos o lo necesitamos en El Salvador actual?

MEV: Sí, efectivamente. Hay que decir que el término belleza también tiene connotaciones culturales, y para el Occidente también tiene que ver con la belleza interior, la virtud. $\mathrm{Y}$ a eso se refiere también el representante del Ministerio de Educación de China. Entonces sí, definitivamente la capacidad integradora que tienen las artes para hacernos conocer nuestra humanidad, para ayudarnos al autoconocimiento, y a través de ello entender la humanidad de los otros, es uno de los potenciales que tiene la educación artística.

Al final, la escuela lo que quiere lograr es una formación integral, que atienda todas las dimensiones humanas. Y si dejamos fuera las humanidades, entre ellas las artes, entonces estamos perdiendo una valiosísima oportunidad que nos da el currículo nacional de primaria de que nuestros alumnos, nuestra niñez, crezca y se desarrolle tanto cognitiva como afectivamente, que desarrolle competencias para la vida.

La educación estética y cultural nos da la oportunidad de desarrollar la capacidad creativa, las capacidades de trabajar en equipo, de leer subtextos, de entender al otro; y de una manera especial, nos permite sentir, saber, cómo podemos aportar a esta realidad que nos enseña, que se resume en la cultura, cómo podemos construir esa cultura.

Cuando la niñez aprende a ver su mundo, a sentirlo, y sobre todo a comprenderlo, entonces tiene la posibilidad, durante el resto de su vida, de asumir protagonismo. Esa construcción ciudadana es parte de lo que se ha quebrado y se ha perdido en el tejido social en El Salvador.

MZ: Sara, ¿cómo ve usted este punto?

Sara Boulogne (SB): Tengan muy buenas tardes. Antes de empezar, agradecer su presencia, y agradecer a Marta Eugenia por la invitación para compartir con ella. Es bien difícil para mí hablar después de ella, porque casi que lo dice todo. Pero voy a tratar de dar un aporte.

Mario hizo esa acotación sobre esa frase, y mientras estaba pasando el video yo la anoté aquí: "Encontrar la belleza, apreciar la belleza y después crear". Creo que con la educación artística, yo cambiaría la palabra belleza, porque la belleza es relativa. Yo diría encontrar los sentimientos y las emociones, apreciarlos, y a partir de eso poder crear.

Si nosotros no estamos en contacto con nuestras emociones, con esa sensibilidad, difícilmente nos vamos a poder expresar y vamos a poder crear. A partir de esa conexión emocional nosotros definimos qué 
es bello, no sólo por la parte estética, sino por las vinculaciones emocionales que tenemos con las cosas y con las personas.

Por allí iría yo. De todo lo demás, no tengo qué agregar.

Pero en lo de encontrar la belleza y apreciarla, sí creo que lo primero es tomar contacto con las emociones y los sentimientos, y la educación artística nos provee de las herramientas para hacerlo.

MZ: En lo que opinan nuestras ponentes, pienso que todos estamos de acuerdo, pero surge también algo que merece ser discutido. $Y$ es que estos objetivos y bondades de la educación artística, entendemos que estarían incluidos como ejes transversales de la educación. Entendemos que la educación en general, y específicamente la educación primaria, debería de permitirnos desarrollar estas competencias. Pero nos encontramos con la paradoja de que la educación artística, en apariencia, no es central.

Veamos. Hablemos de mitos, de mito en un mal sentido, mito en sentido de mentira (el mito filosóficamente es algo muy valioso para la humanidad). Hablemos de creencias falsas y muy extendidas. Quisiera preguntarles: ¿la educación artística es una materia tan básica como las llamadas materias básicas?

MEV: Yo creo que esto da una oportunidad para que todos pongamos en perspectiva precisamente ese mito. He escuchado al ministro de educación mencionar eso. En realidad, las materias son todas básicas en el currículo nacional, porque una razón de ser de ellas es generar el desarrollo integral de la niñez. Y lo fundamental de la educación artística, a diferencia de las otras asignaturas, es que aporta esa capacidad integradora.

La cultura es lo que realmente construye en los humanos, y allí, en la cultura, está depositado todo: los deportes, las aficiones, las relaciones humanas, tanto entre los individuos como entre los grupos, etc., y la educación artística aporta una forma de integrar la vida, una forma de integrarla dentro de todas estas dimensiones y procesos.

Y eso es aprendido: saber entender las distintas facetas de la vida, saber identificarme con una cultura, eso es algo que se desarrolla a través de un proceso formativo, que tiene que empezar desde la niñez.

Entonces, la educación artística, a través de los lenguajes artísticos, tiene una opción muy potente para poder desarrollar esa alfabetización estética y cultural que necesita la niñez, para generar ciudadanía y también un compromiso para constituir nuestra cultura local. Y al mismo tiempo, para desarrollar los potenciales humanos, porque hay una tarea que hacer cuando uno está creciendo. Entenderse a uno mismo, poder entender todas las dimensiones y momentos a los que nuestra propia humanidad nos lleva, eso, en la medida en que lo vamos logrando, nos lleva a entender mejor la humanidad de los otros. Esa es la apuesta. Y los lenguajes artísticos, viniendo desde lo más hondo de la humanidad de los artistas, tienen grandes mensajes y un gran potencial para generar 
ese aprendizaje, ese desarrollo integrador que esperamos en la niñez y la juventud.

MZ: Creo que al ver este punto de las materias básicas, hay que recordar de dónde viene la palabra "básico": base. Y un comentario como este nos sirve para ponernos en perspectiva de que no hay materias a medias, no hay materias que son un poquito menos materias que otras. Si están allí, como ya señala Marta Eugenia, es por un motivo pedagógico, formador, humanizador, vital. Sí, Sara.

SB: Marta Eugenia dijo: "Los lenguajes artísticos vienen de lo más profundo del ser". ¿No es eso básico? O sea: ¿cómo podemos decir que la educación artística no es básica si parte de lo más profundo del ser, si es la expresión pura de alguien? ¿Cómo no pueden considerar esa materia como básica?

Entonces la pregunta surge, a mi criterio, del contexto en el que vivimos: de quién dijo y por qué dijo que esas materias eran básicas y estas no. Recordemos que el sistema educativo responde a una serie de necesidades político-sociales, ¿verdad? Y a veces, esas necesidades no están en consonancia con nuestras realidades, sino que son impuestas.

¿De dónde nacieron esas materias básicas? ¿Por qué no ha cambiado la visión de que esas son las básicas, y no los lenguajes que vienen de lo más profundo de nosotros?

Para mí ese sería el cuestionamiento para los dirigentes y los que hacen las políticas educativas: ¿por qué creen ustedes que la expresión de lo más profundo del ser no es básico, si eso es lo primero que tenemos que entender y hacer crecer?

MEV: Y agregaría que no es sólo la expresión, sino que es la construcción humana que está en juego allí. Y si la ensayamos desde la niñez, tenemos más oportunidad de que en la adultez todos tengamos esa madurez como humanos, y podamos tener también ese compromiso como parte de un colectivo que comparte cultura, hace cultura y construye ciudadanía.

MZ: Quisiera retomar un fragmento del libro de Marta Eugenia, La Educación Artística en la Enseñanza Básica en El Salvador, donde cita al Dr. Picardo. Y dice aquí el Dr. Picardo: “¿Cómo se construyen las realidades y/o significados de los niños y jóvenes en nuestros ambientes escolares? ¿Coexisten en nuestras aulas un pensamiento lógico científico y otro artístico cultural? ¿La educación artística es un adorno o estorbo curricular? ¿Educamos para la identidad o para pasar materias? ¿Poseen nuestros docentes una buena teoría explicativa y aplicable de cómo aprenden los niños?". Y añade Marta Eugenia: "A estas preguntas hay que agregar: ¿los docentes dominan el campo de la Educación Artística bajo parámetros educativos contemporáneos como para generar aprendizajes significativos en dicha área?".

6 Valle Contreras, Marta Eugenia. (2011). La Educación Artística en la Enseñanza Básica en El Salvador. Antiguo Cuscatlán: Centro de Investigaciones en Ciencias y Humanidades (CICH)/Editorial Delgado. Universidad Dr. José Matías Delgado. P. 175. 
Aquí quisiera que pasáramos, ya que hemos hablado del qué de la educación artística, una definición y qué implica, al cómo. Esta educación artística que en el país ya ha llegado a ser entendida como necesaria, ¿cómo debe ser? ¿Qué características debe tener?

MEV: La educación artística que necesitan dominar los docentes es una educación que aproveche los avances internacionales en educación artística, porque, al igual que en educación en general, han habido avances que nosotros, como país, no podemos desaprovechar. Hacer eso sería como negar que se ha avanzado en general en educación, y negarnos la oportunidad, por ejemplo, de trabajar bajo un enfoque constructivista y por competencias, que han sido grandes logros de la educación contemporánea.

Entonces, así mismo, en educación artística ha habido avances internacionales, y los grandes consensos en los foros que se han dado, regionales y mundiales, son grandes líneas, que deberíamos tomar en cuenta para crear una propuesta formativa para docentes en el país.

Una de estas líneas es que la educación artística debería ser pluralista, en el sentido de que tiene la capacidad de absorber, aprovechar, capitalizar tanto los saberes locales y tradicionales, como lo internacional y lo contemporáneo, lo que vendría a ser otra de estas características, de estas grandes líneas que debería tener la educación artística actual en el país: que sea contemporánea.
Y me detengo en particular en esto porque es allí donde podemos ver este tipo de educación con un enfoque contemporáneo, que permite y demanda del docente que tome en cuenta todas las capacidades del educando, incluidos sus presaberes, y, al mismo tiempo, que capitalice todas las propuestas locales y las internacionales.

Y una característica más que la educación artística contemporánea nos pide: que en el entorno escolar no se trabaje únicamente con una sola área artística, porque entonces eso no sería educación artística, estética y cultural, sino que llevaría el sesgo del conocimiento de una sola disciplina artística. Así es que este eje de la educación artística contemporánea nos demanda que el educador tenga la capacidad de trabajar más de un lenguaje, para que al final la niñez en ese entorno escolar vea, empiece a ver, el dibujo de la riqueza que puede aportar la apreciación estética y cultural de la vida.

MZ: Quisiera que Sara nos compartiera su visión de esta pregunta: ¿cómo debe ser la educación artística que se necesita?

SB: Amarrando con lo que dijo Marta Eugenia, tiene que ser multimodal, plural, multidisciplinaria; que no se enfoque en una sola de las artes, aunque la persona más adelante sí se especialice en un área, sino que a nivel de educación Básica se logren experimentar varias disciplinas.

También tiene que ser diversa. Y es que no todos son iguales; no todos van a tener la 
habilidad para dibujar, por ejemplo. No les podemos pedir a todos los niños que dibujen perfectamente un bodegón. Quizá hay un niño o una niña que va a tener una habilidad para hacer cosas tridimensionales y no para el dibujo. Hay otro que se va a expresar más fácilmente a través de las artes escénicas, y dentro de las ellas, hay que diferenciar entre el lenguaje de la danza y el del teatro, que son similares, pero no iguales.

Entonces, esa calidad de plural, de multimodal, tiene que ver con que somos diversos, distintos. ¿Por qué no darles más herramientas a los niños para poder expresarse? Que conozcan lo más que puedan, y cuando ya tengan una idea clara sobre dónde se quieren desarrollar o hacia dónde sus competencias están más enfocadas, que investiguen y profundicen en esa área.

Con respecto a por qué tiene que ser multimodal (de muchas disciplinas y no sólo una), y en cuanto a lo que se está haciendo actualmente, el Ministerio de Educación lanzó en 2015 el Programa de Formación de Docentes en Servicio. Este programa va a durar cuatro años, del 2015 al 2019, y, como decía el Dr. Picardo, es con el afán de mejorar nuestra planta docente a nivel público, actualizar sus competencias en todas las asignaturas. Por supuesto, empezaron con las otras asignaturas. Pero ahorita ya arrancamos con Artística y Educación Física.

El programa está constituido por ocho módulos, para formar a estos docentes durante dos años. No es una capacitación de "hacer un dibujito y ya estuvo"; es una transformación de paradigmas y enfoques hacia esta nueva visión contemporánea de la educación artística.

Actualmente llevamos desarrollados cuatro módulos con los docentes especialistas. Este grupo de especialistas, luego va a replicar esta formación con los docentes de las escuelas. Vamos con el módulo 4 de especialistas y con el módulo 1 de docentes.

Y evidentemente este programa de formación fue diseñado originalmente por un grupo de expertos salvadoreños, entre ellos: Marta Eugenia; Aída Bernal, que está por aquí; Marta Rosales, que estuvo encargada de la parte de música, y Mariem Pleitez, en la parte de danza. Ellas cuatro fueron las propiciadoras para este cambio de la educación artística, y luego también yo, le he dado continuidad con otro grupo de compañeros artistas. Precisamente ahorita los docentes están teniendo un choque fuertísimo. Primero era el cuestionamiento de: "i¿Y cómo vamos a hacer para dar [enseñar] todo, si yo sólo soy músico?!” o “¡Yo soy plástico, yo soy plástico! ¿¿Yo por qué voy a saber de danza?!”.

$Y$ ha costado un poco romperles el cascarón: "Mire, no tenga miedo. Si lo que va a hacer nada más es aprender otro lenguaje. Eso le va a dar más herramientas en el aula". $\mathrm{Y}$ tratar de romper esos bloqueos mentales que uno se pone: "No, yo no puedo bailar". "¡Como n’ombre! ¡Sí puede! Quítese el miedo". Porque si usted tiene miedo - y esto lo hemos hablado con los docentes-, usted 
les está pasando esos miedos a sus estudiantes. Entonces, sáquese el miedo. Diga: "Yo voy a hacer lo mejor que yo puedo". Y el niño va a decir lo mismo: "Yo voy a hacer lo mejor que yo puedo". En ese buscar se va a descubrir.

Entonces la formación docente está ahorita en ese proceso de cambio, en ese proceso de actualización del que nos hablaba el video. Un proceso que ha sido traído por la gente que ha viajado, y que ha tenido estudios fuera, y que ha dicho: "N'ombre, si afuera están pasando otras cosas. Hay que llevar un poquito de eso al país".

MEV: Gracias, Sara. Y agregar a eso que usted dice, vinculándolo con una cuestión que no quisiera dejar pasar. Y es el hecho de que en el currículo salvadoreño conservamos la asignatura de Educación Artística. Lo mismo han hecho los países desarrollados. Para grandes economías, como por ejemplo Singapur, es parte de su triángulo de grandes ejes de la formación en el currículo nacional de educación básica y media. Las artes están allí, y las Humanidades. Y lo mismo sucede en los Países Bajos y Finlandia. Así que eso tiene una razón de ser.

Además, la propuesta de educación artística multidisciplinaria o multimodal es la clave para el país. Con ella, que hace posible la integración a través del lenguaje de las artes, tenemos la oportunidad de aprovechar el valioso tiempo de los alumnos — con dos horas de Educación Artística a la semanaen esa etapa tan crítica.
Y posteriormente, esa avanzada importante: la formación docente en educación artística con enfoque contemporáneo. ¿Por qué digo avanzada? Porque luego de la formación de los docentes viene, con la apertura del Instituto Superior en Artes (ISARTES), que es nacional, el desarrollo de profesionales que son artistas, que son especializados en sus disciplinas, pero además son pedagogos.

$\mathrm{Y}$ entonces, fuera del tiempo curricular, los centros deberían de ir creando clubes, y allí sí hay una identificación de talentos y un cultivo de estos talentos, para que las personas que sí se vayan perfilando con intereses particulares en algún área artística puedan optar por la formación disciplinaria en estos clubes, que deberían de estar en el tiempo extracurricular.

Pero la educación estética y cultural, para tener un desarrollo integral, para desarrollar competencias para la vida, esa es necesario que se genere a través de una formación que enfatice lo estético: la alfabetización estética y cultural. Ese es el motivo de esta propuesta de formador multidisciplinario, multimodal, para la escuela básica.

MZ: Ahondemos un poco en este concepto de la formación multidisciplinaria de los docentes. Y para esto quisiera mostrarles un video del Dr. Ricardo Marín Viadel, que es el coordinador de Facultad de Bellas Artes de la Universidad de Granada, España, es catedrático de Educación Artística en el Profesorado de la Facultad de Educación de esa misma universidad y fue el director de 
tesis doctoral de Marta Eugenia. Él, como una participación en este conversatorio, grabó una serie de videos para describir su visión del trabajo de Marta Eugenia, y yo quisiera que viéramos uno de ellos, a propósito de esta propuesta de formación multimodal del docente.

Dr. Ricardo Marín Viadel: [El discurso viene del video anterior de la serie grabada por el Dr. Marín Viadel]. Una de las consecuencias de esta relación, de esta interrelación mutua entre la Universidad de Granada y la Universidad Matías Delgado, fue que Marta Eugenia se propusiera desarrollar su tesis de doctorado, para obtener el título de doctora por la Universidad de Granada. Y nos pusimos a la tarea. En este caso ella ya había desarrollado previamente diferentes publicaciones en las que está su interés. Por ejemplo, su historia y análisis de la Educación Artística en El Salvador, que es una aportación extraordinaria. $\mathrm{Y}$ en el caso de su tesis doctoral, está enfocada a una investigación en la práctica, en la que ella analiza cuáles eran y cuáles siguen siendo los problemas, la situación y los contextos de la educación artística en El Salvador. Y propone, en conclusión, un proyecto de formación del profesorado, un proyecto interdisciplinar que permitiría implementar todas las energías de los diferentes profesionales y de los diferentes proyectos que ya se estaban llevando a cabo en El Salvador, de una manera sistemática, de una manera realmente coherente, e incorporando las nuevas ideas: posmodernidad, multiculturalismo, nuevas tecnologías, tanto en el ámbito de las artes visuales como en el de las artes escénicas: la danza, el teatro, la música, de manera que se puedan lograr títulos universitarios del más alto nivel, para la formación especializada del profesional de educación artística para los diferentes niveles.

MZ: Bien, este es el comentario del Dr. Marín Viadel sobre la formación interdisciplinar y sobre el trabajo que ha estado realizando Marta Eugenia referente a esto. Ahora, quisiera dar una última mirada sobre este punto, porque pienso que aquí hay algo muy valioso, un par de palabras clave que vale la pena tener claras. Y es que, como se desprende de esta exposición, la educación artística según el enfoque contemporáneo no está orientada directamente a la creación artística. El objetivo de esta educación artística contemporánea, no es que yo como estudiante salga sabiendo pintar o tocar un instrumento musical.

¿Cuál es el paradigma al que esta educación artística contemporánea se opone, y cuál sería, les pregunto de nuevo, el objetivo de esta educación artística? Quisiera aclarar esos puntos.

SB: Como decíamos al inicio, la educación artística actual, la contemporánea, que tiene el carácter de ser multidisciplinar (multimodal), tiene como objetivo no el producto final, no el producto técnico per se, como era antes la educación artística: "Agarre los lápices de colores y aprenda a pintar". Y entonces venía: "¡No se salga de la línea! ¡Coloree para un solo lado!". Esa era la educación artística que nosotros conocimos, que yo conocí: el aprendizaje de las técnicas, y el ser maestro 
en una técnica. Eso tiene que ver con el producto artístico final, con su calidad.

La educación artística contemporánea, estamos hablando de niños, no de profesionales adultos artistas —esos sí se tienen que dar duro con la técnica y perfeccionar su oficio-; los niños, en la actualidad, y por ende los docentes que van a enseñar, tienen que enfocarse en la sensibilización, en el saber expresar, en el saber escuchar, entender. Para que uno pueda crear, primero hay que encontrarse.

Entonces la educación artística actual, la contemporánea, va para allí: a encontrarse uno; luego, va para apreciar lo externo, al otro; luego, de dónde vengo, qué es lo que se genera a mi alrededor, cómo puedo contribuir yo a eso que se está generando alrededor mío. Es más integral; no tiene que ver sólo con la producción final de un objeto o de un resultado, sino con todo el proceso que va involucrado.

MEV: Así es, Sara. Gracias.

Para reforzar esta parte de cómo se ensaya humanidad, las artes y el lenguaje de las artes le permiten al niño empezar a ensayar eso que lo hace humano, eso que lo hace creativo, que lo hace generador de su propio bienestar interno, y también cómo puede ver su realidad y tocar esa realidad para transformarla. Esos mecanismos no son innatos, hay que aprenderlos, y las artes permiten su aprendizaje. Y la sensibilización hacia las artes, al hacer arte y también al apreciar lo que los artistas hacen, nos enseña todas esas rutinas cognitivas que llevan a practicar, a ensayar esa humanidad.

Al final tenemos la posibilidad de que cada quien se encuentre, en ese encuentro personal que nos da el espacio de las artes, el aprendizaje de las artes. Y entonces podemos legar y empezar a aproximarnos a la humanidad de los otros y al sentido de la cultura.

Otra cosa importante es que el arte nos enseña también a dar significado a lo que estamos viviendo, y nos enseña el pensamiento crítico, que es parte de lo que se necesita ahora que estamos tan bombardeados por toda la publicidad y el consumismo. Realmente vemos la necesidad que hay de desarrollar un pensamiento crítico sobre todo lo que nos ofrece la cultura.

También, hay que tener en cuenta que no estamos hablando del término del siglo XVIII de "las bellas artes", esa clasificación tradicionalista, sino que estamos hablando del concepto de arte contemporáneo: las artes están en todos lados; incluyen las artes populares, el diseño, la creación objetual en sí misma, generada por la creatividad humana, y que incorpora también la tecnología. Así que todo ese mundo hay que aprender a verlo, y no darlo por sentado. Y eso se aprende en la educación artística en la enseñanza básica. Por eso hablamos de educación artística, estética y cultural.

MZ: Muchas gracias. Quisiera que tocáramos un último punto, acerca de los desafíos de la educación artística en la formación docente. 
Hemos hablado ya del recorrido que se ha hecho para que la importancia de la educación artística sea evidente. Siempre ha sido importante, pero no era evidente. Como hemos visto, desde el 2008 se han hecho grandes esfuerzos para que se visibilice; se ha logrado una conciencia, sobre todo en los tomadores de decisiones educativos; se están realizando acciones como las que ya se mencionaron, y vamos a la parte que nos falta.

Entendemos que estos planes, como ya lo dijo Sara, no son soluciones a corto plazo, no son parches, sino que buscan ser soluciones que incidan a nivel estructural. Entonces, quisiera preguntar: 1) ¿qué desafíos tenemos? y 2) con base en sus investigaciones y en los programas en los que participan, ¿cómo visualizan a los docentes que se están formando en un mediano plazo, digamos en cinco años?

SB: Creo que, como ya lo hemos dicho, tenemos una serie de desafíos enormes. Porque este rescate que se está haciendo, esta visibilización de la educación artística, es relativamente joven, es reciente. Entonces nos falta saltar un montón de obstáculos. Entre esos obstáculos y desafíos, yo tengo señalados algunos bien específicos.

Si todo esto se está dando, toda esta actualización y puesta en escena de la educación artística a nivel educativo, es porque hay presiones políticas externas. Eso no lo podemos negar. Todo eso que está pasando afuera presiona también al país para que se mueva sobre ese punto. Por lo tanto, uno de los desafíos más grandes es mantener la voluntad política, para que se le siga dando énfasis, para se sigan dando los fondos y el apoyo para el desarrollo de la educación artística de manera seria y comprometida. Y por largo tiempo.

Luego, el correcto desarrollo de los programas de formación docente. Tenemos el gran problema de que en el país no tenemos suficiente gente preparada de manera académica en las áreas de formación artística. Entonces esos recursos son limitados, tanto en personal como en recursos materiales. Ese es otro desafío, que se puede superar. Se está superando desde el momento en que vamos trabajando en eso; vamos caminando.

Hay una frase que me gustó mucho de la Revista de Humanidades y Ciencias Sociales, en la que hay un artículo de Marta Eugenia ${ }^{7}$, donde ella dice que para resolver hay que imaginar, formular, planificar, trabajar y perfeccionar... Es un proceso. Nada se compone, después de haber estado descuidado, de la noche a la mañana. Entonces yo pienso que el desafío más grande que tenemos en la educación artística es la constancia. Porque ya imaginamos; imaginar no cuesta. Ya se formuló, y la formulación ya llegó a ser constante. Estamos empezando la fase de

7 Valle Contreras, Marta Eugenia. "Pensar la educación artística en el currículo nacional: evidencias, reflexiones para imaginar un nuevo escenario de la asignatura en El Salvador". Revista de Humanidades y Ciencias Sociales, 8, segunda época. Jul-dic 2016. Págs. 15-49. Centro Nacional de Investigaciones en Ciencias y Humanidades (CENICH).Ministerio de Educación de El Salvador. 
trabajo. Y para que esta fase se mantenga, hay que darle seguimiento, un buen seguimiento, y fortalecerla. Ya después vamos a ver cómo perfeccionamos.

Por allí está mi visión del proceso.

MEV: Yo me sumo a lo que nos ha dicho Sara. Este proceso pasa por una serie de medidas a corto, mediano y largo plazo, y esperamos que independientemente de los cambios de gobierno, esto se sostenga como agenda de país.

Uno de estos desafíos es tener referentes en el Ministerio de Educación, porque se están tomando decisiones que inciden grandemente en el desarrollo de la educación artística en el sistema educativo, y no hay especialistas de educación artística tomando esas decisiones. Eso complica las cosas. Para tomar decisiones sobre el currículo de Sociales, Matemáticas y las otras asignaturas, sí hay adentro del ministerio especialistas. Entonces, los especialistas que estamos fuera del ministerio, necesitamos un referente dentro del ministerio, y propondríamos la creación de una Coordinación de Educación Artística para hacer una acción afirmativa en este momento en que se necesita ga- rantizar su desarrollo, independientemente del gobierno que esté.

Lo segundo es crear un programa para la apertura de plazas, porque los maestros se pueden empezar a graduar, pero el ministerio debería de ocuparse de ir creando de manera escalonada a la oferta las plazas para colocarlos en el sistema educativo público. Por supuesto, con sueldos dignos que llamen la atención de profesionales en esta área.

Y por último, los recursos. Garantizar los recursos también de manera escalonada, para que cada centro escolar tenga todo el equipo necesario, sobre todo porque estamos llegando a un momento en el que las artes se combinan mucho con la tecnología. Lo vivimos cada día, todos los ciudadanos del mundo. $Y$ eso no es ajeno al mundo de las artes ni a nuestro imaginario de formación estética, ciudadana y cultural.

MZ: Esta, como vemos, es una plática apasionante y amplia, que debe continuar, que los invito a que la continúen, conversando con nuestras invitadas y leyendo los textos de Marta Eugenia.

Agradezco muchísimo sus aportes de hoy. 